\title{
Adverse events of monoclonal antibodies use in therapy of hematological malignancies
}

\author{
Marcela Maksymowicz ${ }^{1}$ (D) Monika Podhorecka ${ }^{2}$ (D) \\ ${ }^{1}$ Student Research Group at the Department of Hematooncology and Bone Marrow Transplantation, \\ Medical University of Lublin \\ ${ }^{2}$ Department of Hematooncology and Bone Marrow Transplantation, Medical University of Lublin, Lublin, Poland
}

\begin{abstract}
Monoclonal antibodies given as monotherapy or combination therapy have emerged as effective treatment options for hematologic malignancies. By prolonging survival, mAbs reduced mortality and improved the clinical prognosis for patients with these diseases. However, despite the effective anticancer activity of $m A b s$, they induce adverse events. The most common side effects are infusion related reactions (IRR), associated with cytokine release within the first few hours after administration. IRR are usually mild to moderate and manifest in rash, fever, nausea, vomiting, dizziness, headache, hypotension or tachycardia. Other, common toxicities are cytopenias, increasing the risk of infections and bleeding. Most preventive strategies involve the use of glucocorticosteroids, acetaminophen, antihistamines, screening for antibodies against microorganisms and prophylaxis for infections. Cytokine release syndrome, cardiac, pulmonary, neurologic adverse effects occur less frequently. In cases of grade 1-2 toxicity, symptomatic management is recommended, but in more severe symptoms temporary or permanent discontinuation of therapy and use of glucocorticosteroids are recommended. In an effort to limit the incidence and severity of adverse events clinicians should know how to early recognize, precisely assess and timely manage.
\end{abstract}

Key words: monoclonal antibodies, hematological malignancies, adverse effects

Hematology in Clinical Practice 2021; 12, 3-4: 121-131

\section{Introduction}

Over recent years, tremendous progress in identifying therapeutic targets in hematological malignancies has been observed, leading to the discovery of new drugs with effectiveness proven in clinical trials [1]. Monoclonal antibodies (mAbs) bind to specific molecules on immune cells and activate various signaling pathways in the immune system. They may contribute to antibody-dependent cellular cytotoxicity (ADCC) though natural killer (NK) cells, antibody-dependent cellular phagocytosis (ADCP) though macrophages, or complement-dependent cytotoxicity (CDC) [2]. $\mathrm{mAbs}$ have revolutionized the treatment of hematological malignancies improving clinical outcomes. Nevertheless, this rapid development of therapy is accompanied by toxic effects, most of which are interdisciplinary in nature and a challenge for both hematologists and intensive care physicians [3]. Biomarkers of adverse events (AEs) induced by $\mathrm{mAbs}$ are not identified, therefore clinicians of all disciplines should be aware of the toxicity associated with mAbs therapy. This will increase the chances of effective outcomes optimization with the use of these agents in hematological malignancies.

Address for correspondence: Marcela Maksymowicz, Klinika Hematoonkologii i Transplantacji Szpiku, Uniwersytet Medyczny w Lublinie, ul. Staszica 11, 20-081 Lublin, Poland, phone +48 81534 23 97, fax +48 815345605 , e-mail: marcela.maksymowicz@gmail.com

This article is available in open access under Creative Common Attribution-Non-Commercial-No Derivatives 4.0 International (CC BY-NC-ND 4.0) license, allowing to download articles and share them with others as long as they credit the authors and the publisher, but without permission to change them in any way or use them commercially. 


\section{Rituximab}

Rituximab (RTX) is a monoclonal antibody directed against the CD20 antigen present on B lymphocytes surface, indicated in the treatment of B-cell lymphoma, lymphoproliferative disorders and some autoimmune diseases [4]. Infusion related reactions (IRRs) are commonly reported AEs following the use of RTX. In clinical trials, they were reported in $77 \%$ of patients with nonHodgkin lymphoma (NHL) and chronic lymphocytic leukemia (CLL) [4]. Intravenous administration of RTX has been associated with reactions such as rash, fever, nausea, vomiting, dizziness, headache, hypotension and tachycardia. Therefore, the infusion should be performed slowly and the patient's condition should be monitored during and after completion of infusion [5]. To reduce the risk of RTX side effects, premedication should include glucocorticosteroids, paracetamol, and an antihistamine [4]. Most of the IRRs were recorded during the first RTX infusion within 1-2 hours of the infusion, and their incidence decreases with subsequent infusions. B-cell targeted therapies, including RTX, contribute to a B-cell reduction, hypogammaglobulinemia and an increased risk of infections [3, 6]. Hepatitis B virus (HBV) reactivation induced by combining RTX with chemotherapy has been reported, therefore, screening for chronic and previous HBV infection, including hepatitis $\mathrm{B}$ surface antigen (HBsAg) and antibodies against HBV should be performed. Other infections seen in studies were exacerbations of hepatitis $\mathrm{C}(\mathrm{HCV}$, hepatitis $\mathrm{C}$ virus), herpes virus infections, including herpes zoster virus (HZV), human herpes virus 3 (HHV-3), varicella zoster virus (VZV), as well as progressive multifocal leukoencephalopathy (PML). According to the European Conference on Infections in Leukemia, prophylaxis against Pneumocystis jiroveci pneumonia (PJP) is recommended in patients undergoing R-CHOP therapy (RTX, cyclophosphamide, doxorubicin, vincristine and prednisolone) [6]. For this reason, administration of immunoglobulins is also indicated in most patients receiving RTX and other anti-CD20 drugs in the treatment of B-cell neoplasms [3]. The mechanism of action of anti-CD19 therapy is similar to that of anti-CD20 antibodies, so their use also requires immunoglobulins substitution [3]. In studies involving NHL and CLL patients, combining RTX with chemotherapy was not associated with an increased incidence of infections, but with higher risk of grade 3-4 hematological complications - leukopenia, neutropenia and pancytopenia, compared to chemotherapy alone [4]. However, age $\geq 70$ years was a risk factor for severe hematological AEs and bacterial infections in patients with previously untreated or relapsed/ /refractory (R/R) CLL treated with RTX in combination with chemotherapy. Late-onset neutropenia is also possible - up to 5 months after the end of therapy in patients treated with RTX [6].

Apart from IRR and infections, RTX can cause mucocutaneous complications, arrhythmias, renal and gastrointestinal dysfunction [5]. Decrease in the number of CD20+ lymphocytes can cause ileitis, as their presence in the gut is considered a protective factor and potentially preventing inflammation [7]. Therefore, therapy with anti-CD20 RTX may lead to dysregulation of T-reg lymphocytes and autoreactive stimulation of $\mathrm{T}$ lymphocytes, contributing to ileitis or exacerbation of inflammatory bowel disease (IBD).

An alternative to the intravenous form of RTX is subcutaneous RTX [5]. This new form of the drug has been approved by the US Food and Drug Administration (FDA) for the treatment of adults with follicular lymphoma (FL), a diffuse large B-cell lymphoma (DLBCL) and CLL. Compared to intravenous infusion, the advantage of this therapy is the reduction of the time of drug administration, time spent in the clinic and increased patient comfort [5]. It has been shown that AEs resulting from subcutaneously administered RTX in patients treated for FL, CLL or DLBCL were similar [5]. Due to lower costs compared to the original drugs, equivalent biological drugs biosimilars have become more and more widely used, including RTX biosimilar [8]. In a cohort study with NHL and CLL patients, the incidence and severity of AEs were similar for both formulations.

\section{Obinutuzumab}

Obinutuzumab is also an anti-CD20 antibody with the potential to overcome the mechanisms of RTX resistance. However, this drug is considered to be more toxic than RTX [9]. Irina Amitai et al. meta-analysis reported an increased risk of grade 3-4 AEs, including infections, IRR, thrombocytopenia and cardiac events after the use of obinutuzumab, compared to RTX [10]. The risk of infection is increased by the underlying disease, comorbidities that are common in the elderly, and the chemotherapy regimen used. Despite the high risk of infectious disease, there was no difference in the incidence of severe neutropenia (grade 3-4) between RTX and obinutuzumab. Obinutuzumab 
in combination with venetoclax in CLL therapy is associated with grade 3-4 neutropenia with a frequency of 53-73\% [11]. The development of thrombocytopenia and IRR is associated with the release of interleukins (ILs): IL-8, IL-6 and tumor necrosis factor alpha (TNF- $\alpha$ ) as well as with cytokine release syndrome (CRS) [10]. Severe CRS, observed in patients treated with obinutuzumab, results in a higher rate of cardiotoxicity compared to RTX. In addition, the use of obinutuzumab with chemotherapy has been associated with the development of secondary hematological malignancies [10].

\section{Brentuximab vedotin}

Brentuximab vedotin (BV), mAb conjugated with monomethylated auristatin $\mathrm{E}$ (MMAE), a very potent anti-microtubule agent, directed against the CD30 receptor, is used in the treatment of classical Hodgkin lymphomas (cHLs) and peripheral T-cells lymphomas [12]. In the treatment of lymphoma patients, BV increased the risk of severe AEs in the form of peripheral sensory neuropathy, nausea, vomiting, and diarrhea. Peripheral neuropathy, a common side effect of BV (observed in about $60 \%$ of patients) manifested by numbness and tingling in the limbs, is induced by the toxic effect of MMAE on axonal microtubules [13]. It is also possible to develop neuropathy with immunological pathogenetic factors. Although the use of BV in combination with standard chemotherapy (ABVD: adriamycin, bleomycin, vinblastine, dacarbazine) in the first-line treatment in advanced cHL resulted in a high percentage of complete remission (CR), a high percentage of pulmonary toxic effects was also observed [14]. Studies show, that limiting co-administration of bleomycin with BV reduces the risk of pulmonary toxicity. However, BV combined with AVD resulted in a higher rate of neuropathy and neutropenia compared to BV-ABVD [15]. In order to prevent hematological complications, especially neutropenia associated with BV + ABVD therapy, administration of granulocyte colony stimulating factors (G-CSFs) is recommended. In addition, prevention of cytomegalovirus (CMV) infection by viral prophylaxis or plasma CMV PCR testing should be considered in patients receiving BV.

$\mathrm{BV}$ can be used in combination either with chemotherapy, or with immunotherapy. In phase II study with combination of two new drugs - BV and nivolumab - in the treatment of R/R HL patients, peripheral neuropathy was a troublesome side effect [16]. Neurological symptoms can be related to neuropathy, but also to PML. For this reason, in the case of neurotoxicity symptoms, in-depth differential diagnosis is essential. Exacerbation of neurotoxicity may lead to treatment discontinuation, so careful observation of behavior and neurological signs and symptoms in patients undergoing therapy is indicated [6].

\section{Blinatumomab}

Blinatumomab, as a bispecific $\mathrm{mAb}$, binds to the CD3 + T-cell and the CD19+ B-cell, leading to T-cells activation as well as B-cells apoptosis and lysis [17]. The release of perforin and granzymes from granules in cytotoxic T-cells induces cytotoxicity of target B-cells and therefore is applicable in $\mathrm{R} / \mathrm{R}$ therapy of acute lymphocytic leukemia (ALL). Antigen-antibody interaction stimulating T-cell activation induces toxic effects. The major, serious AEs of blinatumomab in R/R ALL therapy are neurological adverse events (NAEs) and CRS.

Antibody targeting to CD19 molecules is presumed to be the cause of neurotoxicity due to similar symptoms in patients treated with T-cells with chimeric antigen receptors cell therapy (CART) targeting CD19 [17]. The neurological toxicity observed with blinatumomab treatment is believed to be related to the production of neurotoxic cytokines and chemokines upon activation of T-cells, which leads to irritation of the neurendothelium.

It is assumed, that antibody targeting CD19 molecules causes neurotoxicity due to similar symptoms in patients treated with T-cells with CART targeting CD19 [17]. The neurological toxicity observed with blinatumomab treatment is believed to be related to the production of neurotoxic cytokines and chemokines upon T-cells activation, which leads to irritation of the neuroendothelium [18]. Neurotoxicity can manifest as nonspecific symptoms - headache, tremors, confusion or aphasia, convulsions or dementia. In the TOWER study with $405 \mathrm{R} / \mathrm{R}$ ALL patients, the proportion of NAEs of any grade was higher in the blinatumomab group than in the standard of care chemotherapy (SOC) group (61\% vs. $50 \%$, respectively). NAEs were more likely to cause discontinuation of therapy in the blinatumomab arm (6\% vs. $1 \%)$. Occasionally, especially in the case of grade 1-2 toxicity, dexamethasone may reduce the severity of the neurological side effects without discontinuing blinatumomab treatment. In the case of grade 3 NAEs, treatment must be interrupted for at least 3 days, but grade 4 NAE is confirmed, blinatumomab should be permanently discontinued. An important strategy to prevent AEs associated with the use of 
blinatumomab is to gradually increase the dose of blinatumomab from $9 \mu \mathrm{g}$ /day in the first week of therapy to $28 \mu \mathrm{g} /$ day from the second week to the end of treatment.

CRS manifests itself with fever, chills, fatigue, low blood pressure, and symptoms related to capillary leak syndrome (CLS). The incidence of any grade CRS was higher in R/R ALL patients than in MRD-positive ALL patients (16\% vs. $3 \%$ ) due to the difference in tumor burden between groups. Prophylactic management include dose modification, discontinuation of blinatumomab treatment, cytoreduction, and dexamethasone. Additionally, intravenous fluids and tocilizumab, an IL-6 inhibitor, can be used [19]. If grade $3 \mathrm{CRS}$ is diagnosed, blinatumomab treatment may be resumed after discontinuation and administration of dexamethasone. In the case of grade 4 toxicity, treatment discontinuation is recommended [17]. Due to the mass production of cytokines such as IL- $1 \beta$, IL-2, IL- 6 and TNF- $\alpha$, CRS is considered a risk factor for cardiac dysfunction and hemophagocytic lymphohistiocytosis.

The drug targeting CD19 molecules induces decrease of plasma cells and T lymphocytes count, as well as neutropenia [17]. Interestingly, the incidence of cytopenia and serious infections was lower in the blinatumomab group than in the chemotherapy arm (60\% vs. $70 \%$ and $34 \%$ vs. $52 \%$, respectively). Nevertheless, blinatumomab has been associated with catheter-related bloodstream infections, therefore patients receiving the drug should be carefully monitored for signs and symptoms of infection. Moreover, CRS, infections and febrile neutropenia were more common AEs of blinatumomab administered in second or later line of treatment compared to patients treated previously, while neurological toxic effects and neutropenia were more frequent during first-line treatment [19]. Also, serious AEs and treatmentrelated fatal events were more frequently reported in the group receiving blinatumomab as a second or subsequent line of therapy. However, these results may be due to the higher stage of the disease and worse prognosis of patients receiving drug in the later treatment lines.

\section{Inotuzumab}

Inotuzumab ozogamicin ( $\mathrm{InO})$ is a humanized anti-CD22 antibody combined with the alkylating agent calicheamicin used in the treatment of $R / R$ ALL and NHLs. Release of calicheamicin into lysosomes in the cytoplasmic cell leads to double- stranded DNA cleavage and subsequent cell apoptosis [20,21]. However, the direct toxic effect of the antibiotic is suspected of damaging liver cells, contributing to the inhibition of sinusoidal flow. Sinusoidal obstruction syndrome (SOS) is a serious complication after allogeneic stem cell transplantation (allo-SCT) and a symptom of hepatotoxicity [17]. It is believed that the higher incidence of SOS in pediatric patients (under 18 years of age) than in adults may be due to incomplete structural maturation of hepatic vessels in infancy. Earlier hematopoietic stem cell transplantation (HSCT), the use of other anticancer drugs and elevated levels of hepatic transaminases [aspartate aminotransferase (AST), alanine aminotransferase [ALT]) before HSCT are risk factors for SOS associated with the use of InO [22]. The clinical classification of SOS includes the level of total bilirubin, liver enzymes, serum creatinine, weight gain and the clinical progression rate [1]. Compared to hyperbilirubinemia and elevated transaminase level, severe SOS has less common and less specific symptoms, such as hypoxia, encephalopathy, and renal failure [17]. A liver biopsy is the best diagnostic method, but may result in bleeding due to thrombocytopenia. Prior to initiating $\mathrm{mAb}$ therapy, risk factors such as previous myeloablative conditioning, older age, and history of liver disease should be assessed. The risk of SOS can be reduced by using fractionated $\mathrm{mAbs}$ and extending the time between the last dose of InO and the HSCT [20].

Additionally, bilirubin and transaminase levels should be monitored during InO therapy. According to studies in which defibrotide improved survival rates in patients with veno-occlusive disease (VOD), it is believed that defibrotide may be an effective agent in the treatment of VOD [21]. In order to reduce the toxicity of the drug, it is worth avoiding nephrotoxic and hepatotoxic drugs such as azoles, prophylactically using ursodeoxycholic acid and considering combination therapy [1]. Consolidation therapy in the form of blinatumomab in the mini-HCVD-INO-blinatumomab regimen may extend the time interval between InO and allo-SCT and further reduce the risk of VOD [23]. While VOD is one of the frequently reported toxic effects of $\mathrm{InO}$, the most frequently reported serious AEs during ALL and NHLs therapy were thrombocytopenia and neutropenia, with at least grade 3 events occurring in $29 \%$ and $48 \%$ of patients, respectively. Interestingly, the incidence of cytopenia was similar during CART therapy (CAR19/22). Neutropenia was reported more frequently in patients with a median age $\geq 60$ years 
or in patients with NHLs. Although the incidence of neutropenia in the treatment of R/R B-cell ALL was also high, infections were less frequently reported in this group of patients than in the group treated with chemotherapy. To prevent cytopenia, it is recommended to monitor complete blood count $(\mathrm{CBC})$ before each InO cycle and react in the case of infection, sepsis or bleeding appearance.

Other AEs associated with InO treatment are QT prolongation and tumor lysis syndrome (TLS) $[17,21]$. Prolonged QT interval may increase the risk of torsades de pointes and sudden cardiac death and therefore requires prompt intervention [1]. Electrolytes and concomitant use of drugs due to comorbidities may also affect the electrocardiogram, therefore special ECG monitoring is recommended when using drugs that prolong the QT interval [1].

\section{Gemtuzumab ozogamicin}

The addition of gemtuzumab ozogamicin (GO) to standard chemotherapy for acute myeloid leukemia (AML) resulted in an increase in event-free survival (EFS), relapse-free survival (RFS), but also reducing the risk of nausea, vomiting, diarrhea and grade $3-4$ hepatotoxicity [24]. GO is a cytotoxic calicheamicin-conjugated recombinant humanized anti-CD33 mAb approved for the treatment of AML. Due to conjugation with calicheamicin, hepatotoxicity is a common $\mathrm{AE}$ of $\mathrm{GO}$, as during InO therapy. VOD, a potentially fatal condition was reported more frequently in AML patients treated with GO compared to R/R B-cell NHL patients treated with InO (9\% vs. $1 \%$ ), but less frequently than in $\mathrm{R} / \mathrm{R}$ ALL patients treated with InO [1]. However, the use of fractionated GO, reduced intensity of conditioning, and maintaining an interval of at least 60 days between the last administration of GO and HSCT may be responsible for a similar incidence of post-transplant VOD/SOS in the group treated with GO combined with chemotherapy compared to the group not treated with GO, which suggests the possibility of using HSCT in the consolidation therapy of AML patients previously treated with GO [25]. In randomized ALFA-0701 study, the main toxicity associated with the use of GO combined with chemotherapy (daunorubicin and cytarabine) was thrombocytopenia, lasting 45 days after starting treatment, reported in $20 \%$ of patients [26]. Treatment of GO is also associated with the risk of myelosuppression, IRR and TLS [27]. Prevention of TLS consists of hydration, use of hypouricemic drugs, renal replacement therapy and correction of electrolyte disturbances. It is important to monitor patient general condition, electrolytes blood levels, and initiate TLS treatment promptly as TLS-related ionic and metabolic abnormalities can result in renal failure, cardiac arrhythmias, and death. In order to increase stability and efficacy of calicheamicin-conjugated antibodies, GO and $\mathrm{InO}$, in the treatment of hematological malignancies, modified conjugates were developed by direct binding of reduced calicheamicin thiol to modified cysteine on the antibody [28]. This is a promising form of therapy due to the fact that the altered drug has been proven to be less toxic in animal studies.

\section{Daratumumab}

Daratumumab is an anti-CD38 antibody used as monotherapy or in combination with traditional treatment regimens for multiple myeloma (MM) in adults $[6,29]$. The most common AEs associated with newly diagnosed (NDMM) and relapsed/ refractory multiple myeloma (RRMM) were IRRs, mostly mild, grade 1-2 and usually occurred during the first administration or within 4 hours of infusion completion [6, 29, 30]. Despite the well-known drugs used in premedication, such as glucocorticoids, antipyretics and antihistamines, according to Chari et al., montelukast, a leukotriene receptor antagonist can significantly reduce the risk of IRR. The use of subcutaneous daratumumab is associated with similar efficacy, safety and a lower IRR prevalence compared to the intravenous form [29, 31].

Other frequently reported AEs were diarrhea, cytopenia and respiratory infections [1, 29]. The hematotoxicity index may increase due to the synergistic effect of concomitantly administered drugs. VZV infection was a more frequent adverse effect in combination therapy than in monotherapy, but the incidence of neutropenia and all infections was similar in both groups [6]. In seropositive patients, infection prophylaxis should be considered.

Daratumumab may induce hematotoxicity via the cytopenia, but it may also disrupt the binding of red blood cells to CD38 [29]. This interaction can lead to general in vitro reactivity, a positive indirect Coombs test (ICT) and a delay in blood transfusion performance. Dithiothreitol (DTT) is added to the blood sample to reduce binding of daratumumab to blood cells, breaking extracellular binding to CD38. Another approach is to neutralize daratumumab with recombinant human anti-daratumumab antibodies, but their use is still limited. In a study 
evaluating the efficacy and safety of daratumumab with bortezomib and dexamethasone $(\mathrm{D}-\mathrm{Vd})$ versus bortezomib and dexamethasone (Vd) in patients with MM first relapse, peripheral neuropathy (50 vs. $38 \%$ for DVd and Vd) and secondary malignancies (6\% vs. $2 \%$ for DVd and Vd) were observed slightly more often.

\section{Elotuzumab}

Elotuzumab, mAb directed against signaling lymphocyte activation molecule family 7 (SLAM F7) in plasma cells is used in the treatment of RRMM. The most serious AEs (grade $\geq 3$ ) following administration of elotuzumab with thalidomide and low dose of dexamethasone were fatigue, peripheral edema and IRR [31]. According to several studies, elotuzumab was less likely to cause lymphopenia in RRMM and was considered less toxic than daratumumab [32]. In therapy with thalidomide and dexamethasone, the most common side effects were fatigue, pulmonary edema and IRR [31]. Elotuzumab in combination with lenalidomide and dexamethasone (Elo-RD) in RRMM therapy reduced the risk of disease progression and death with a similar frequency of toxic effects [33]. The primary grade 3-4 AEs were lymphocytopenia (79\%), neutropenia (36\%), infections (33\%), and thrombocytopenia (21\%). The higher incidence of elotuzumab AEs such as infections and newly diagnosed malignancies is believed to reflect the longer duration of treatment. When elotuzumab was added to $z$ pomalidomide (Elo-PD), therapeutic efficacy was demonstrated with a similar degree of toxicity to Elo-RD therapy. Neutropenia, pneumonia and infections were less frequent, while thrombocytopenia and cardiac events were slightly more frequent. Although elotuzumab is considered an effective treatment strategy for RRMM, there is a need for further studies on the efficacy and safety of combination therapies with various drugs.

\section{Alemtuzumab}

Alemtuzumab is $\mathrm{mAb}$ directed against the CD52 surface antigen, which is expressed on both normal and malignant $\mathrm{B}$ and $\mathrm{T}$ lymphocytes [34]. Its selective action has been used in the treatment of B-CLL, NHLs, T-cell prolymphocytic leukemia (T-PLL), mycosis fungoides (MF) and Sézary syndrome (SS) [35, 36]. Due to the induction of a deficiency of $\mathrm{B}$ and $\mathrm{T}$ lymphocytes, the drug is immunosuppressive, increasing the risk of infection. Immunodeficiency may appear up to 9 months after stopping treatment [6]. Grade 3 lymphocytopenia was the most common hematological toxicity (59\%) of alemtuzumab in a phase II trial in adult patients with T-cell leukemia/lymphoma (ATL), a disease characterized by lymphocytosis [35]. However, infections were less common (14\%), possibly due to $\mathrm{PCP}$, antiviral, and antifungal prophylaxis. On the other hand, alemtuzumab + CHOP used in the treatment of peripheral T-cell lymphoma (PTCL) resulted in serious infections (40\%), including CMV and EBV, despite the applied prophylaxis [37]. Due to the risk of severe immunosuppression and infectious complications, it is recommended to perform antibody screening and appropriate prophylaxis of diseases such as latent tuberculosis, $\mathrm{HBV}$ reactivation, $\mathrm{HCV}$ and opportunistic infections $[6,34,36]$. Further studies are needed to assess whether new drugs used in the prophylaxis of $\mathrm{CMV}$, valganciclovir or letermovir and posaconazole against fungal infections can more effectively prevent the toxicity of alemtuzumab [37]. EBV reactivation and newly diagnosed DLBCL have also been reported in patients with PTCL treated with alemtuzumab, therefore vigilance should be exercised against the development of EBV + DLBCL as a comorbid condition.

The immune system, through the release of cytokines, can lead to a skin rash that can be prevented with antihistamines and paracetamol [34]. Glucocorticosteroids (GCS) are necessary for more severe events and IRRs. CRS with an increased concentration of TNF- $\alpha$, INF- $\gamma$ in the serum and IL- 6 or infiltration of T lymphocytes in the heart may contribute to cardiotoxicity, especially in patients with MF/SS [38]. Despite these known complications, the use of alemtuzumab to prevent graft-versus-host disease $(\mathrm{GvHD})$ may trigger autoimmune reactions such as pure red cell aplasia (PRCA) or autoimmune hemolytic anemia (AIHA) [39].

\section{Mogamulizumab}

Although alemtuzumab and BV appear to be effective therapeutic options for cutaneous T-cell lymphoma (CTCL), there are still studies ongoing on the use of antibodies with more satisfactory efficacy and less toxicity in the treatment of Tcell lymphoma [40]. One of the newest drugs is mogamulizumab, $\mathrm{mAb}$ directed against the $\mathrm{CC}$ chemokine receptor 4 (CCR4). The target of the drug's action is present on the surface of tumor cells in most ATL patients, some patients with other PTCL and CTCL subtypes, but also on the surface of effector T-reg cells, which have the greatest inhibi- 
tory effect on the anti-tumor immune response. The IRR, major AE of mogamulizumab is thought to be due to a defucosylated Fc region on IgG1, which strongly activates NK cells and causes the release of cytokines and cytotoxic molecules [25, 41, 42]. Other common toxicities include rash and hematological complications, neutropenia and lymphopenia, considered as an expected effect related to the therapeutic target of mogamulizumab [25, 40, 42]. Serious dermatological AEs, such as Stevens-Johnson syndrome or toxic epidermal necrolysis, are rare but potentially life-threatening or even fatal. In patients undergoing allo-SCT, the use of mogamulizumab increases the risk of acute GvHD. Therefore, it is recommended to monitor T-reg counts and postpone transplantation for at least 50 days after the last administration of mogamulizumab. This is very important because allo-SCT is an important therapy for both advanced CTCL and ATLL. Serious toxicity with mogamulizumab also includes autoimmune AEs, mostly reversible with glucocorticosteroids. The use of mogamulizumab in patients with autoimmune diseases is relatively contraindicated due to the mechanism of action, T-reg cell depletion, and severe grade $\geq 3$ AEs previously reported, including immune-related myositis, myocarditis, polymyositis, hepatitis, pneumonitis or Guillain-Barré syndrome [41].

\section{Immune checkpoint inhibitors}

Immune checkpoint inhibitors (ICIs) by blocking signaling pathways such as cytotoxic T-lymphocyte-associated antigen 4 (CTLA-4), programmed death protein-1 $[\mathrm{PD}(\mathrm{L})-1]$ molecules, allow anticancer and immune response [6]. Malignant hematological cells may also become targets for antibodies in cancer immunotherapy due to the expression of immune checkpoint molecules on their surface $[43,44]$. The FDA has approved two molecules for the treatment of hematologic malignancies, e.g. nivolumab (anti-PD-1 mAb) for the treatment of $\mathrm{R} / \mathrm{R} \mathrm{cHL}$ and pembrolizumab (anti-PD-1 mAb) for the treatment of $\mathrm{R} / \mathrm{R}$ cHL as well as $\mathrm{R} / \mathrm{R}$ primary mediastinal large B-cell lymphoma (PMBL) [45]. These agents are also promising drugs in the treatment of MM, certain types of NHL, CLL, AML, and myelodysplastic syndromes (MDS) $[45,46]$. However, ICIs mechanism of action, stimulation of the immune system and autoimmunity can lead to immune-related adverse events (irAEs) that can occur in any system and organ in the body, heart, lungs, skin or endocrine system. These toxic effects include myocarditis, pneumonia, kidney or liver toxicity.
The most common side effects of ICIs therapy of hematological malignancies are dermal toxicities. They manifest as rash, dermatitis, erythema nodosum, but can also cause skin necrosis or Stevens-Johnson syndrome. Combination therapy with nivolumab and other targeted drugs increased the risk of serious toxic effects. irAEs affect the balance of the endocrine system and can disturb the functioning of endocrine organs. Thyroid dysfunction was similar for nivolumab and pembrolizumab, and hypothyroidism (0-29\% and $0-17 \%$ ) was more common than hyperthyroidism (0-13\% and $0-17 \%)$. In addition, hyperthyroidism usually progressed to organ failure and required constant hormone replacement therapy. Adrenal insufficiency and type 1 diabetes have also been observed. Gastrointestinal (GI) disorders, the most common of which was diarrhea, were more frequently caused by anti-CTLA- 4 antibodies than by anti-PD(L)- 1 antibodies. A rare but potentially dangerous complication of ICI therapy is cardiotoxicity and pulmonary toxicity, more common with anti-PD(L)- 1 antibodies. Pneumonitis, which may manifest itself even 10-12 weeks after the initiation of ICIs therapy, is the leading cause of deaths associated with the use of ICIs in the treatment of hematological malignancies and solid tumors. In addition, some studies suggest that treatment with ICIs increases the risk of GvHD in patients with bone marrow malignancy undergoing HSCT.

Overexpression of PD-L1 on AML blasts and PD-1 on T lymphocytes has prompted the study of ICIs in some hematological neoplasms [46]. However, increased PD-L1 expression on leukemic blasts may be due to the use of interferon gamma $(\mathrm{INF}-\gamma)$ and induction chemotherapy. The use of ICIs in patients with AML after or before alloHSCT resulted in GvHD, but resolved in most of them after administration of glucocorticoids [47].

ICIs have been used in the treatment of leukemia, lymphoma, but also MM [48]. Compared to patients treated with lenalidomide and dexamethasone, the addition of the third drug, e.g. pembrolizumab to lenalidomide and dexamethasone in first line treatment of MM resulted in a similar frequency of any grade AEs (94\% vs. $92 \%$ ) and a higher rate of serious toxic effects (54\% vs. 39\%). In addition, the risk of death in the pembrolizumab group was higher than in the lenalidomide and dexamethasone group, but these patients were older and had a high cytogenetic risk. This study showed an unfavorable risk profile in patients with NDMM. However, other drug combination regimens, taking into account the patient's clinical 
condition, may increase the efficacy of ICI therapy in hematological malignancies, including MM.

The results of studies with ICIs in the treatment of solid tumors have shown that these drugs cause serious bacterial, viral, fungal infections or PJP. However, it has not been clearly proven whether the use of glucocorticosteroids and infliximab to inhibit the development of irAEs increases the risk of infection [6]. Due to the use of ICIs in combination with chemotherapy, monoclonal antibodies or CART, an increased risk of therapy side effects is suspected. More research is needed on their effectiveness with the lowest possible toxicity.

Immunotherapy with ICIs may be continued in the case of mild grade 1 irAEs [1]. If the toxicity grade is higher, treatment should be discontinued until the toxic symptoms are resolved or permanently. The evaluation and management of irAEs treatment is based on the Common Terminology Criteria for Adverse Event (CTCAE) v5.0. Usually, the use of immunosuppressants like GCS as the first-line drug or infliximab for colitis and mycophenolate for hepatitis results in a reduction in the severity of the toxic effects of therapy $[1,6]$. The management of AEs induced by ICIs and other antibodies used in the treatment of hematological malignancies is presented in Table 1 .

\section{Summary}

Monoclonal antibodies are highly effective in the treatment of hematological malignancies, which is confirmed by the improvement of treatment outcomes and response rates in numerous studies. However, they can cause side effects manifested by infusion-related reactions as well as myelosuppression, infections and autoimmune diseases. While using monoclonal antibodies in hematological malignancies, clinicians should be aware of potential side effects, identify and treat toxic effects as early as possible to mitigate adverse effects of therapy and optimize treatment outcomes.

\section{Conflict of interest}

None.

\section{Funding}

None.

Table 1. The main adverse events of monoclonal antibodies (mAbs) used in therapy of hematological malignancies and prophylaxis and treatment suggestions

\begin{tabular}{|c|c|c|}
\hline Side effect & Clinical manifestation & Prophylaxis and treatment suggestions \\
\hline \multirow[t]{6}{*}{$\begin{array}{l}\text { Infusion-related } \\
\text { reactions }\end{array}$} & \multirow{6}{*}{$\begin{array}{l}\text { Fever, chills, hypotension, } \\
\text { tachycardia, sore throat, } \\
\text { cough, nausea and vomiting } \\
\text { Severe reactions - broncho- } \\
\text { spasm, shortness of breath, } \\
\text { hypoxia and hypertension }\end{array}$} & $\begin{array}{l}\text { - The drug should not be administered to patients with hypersensitivity } \\
\text { to the active substance or any of the excipients }\end{array}$ \\
\hline & & $\begin{array}{l}\text { - Slow drug infusion under close clinical observation, including heart } \\
\text { rate, blood pressure and temperature monitoring }\end{array}$ \\
\hline & & $\begin{array}{l}\text { - The use of premedication - glucocorticosteroids, antipyretic } \\
\text { drugs, antihistamines }\end{array}$ \\
\hline & & $\begin{array}{l}\text { - The addition of montelukast may reduce the risk of adverse } \\
\text { reactions of daratumumab therapy }\end{array}$ \\
\hline & & $\begin{array}{l}\text { - Using an available subcutaneous form of the drug (rituximab/ } \\
\text { /daratumumab) instead of the intravenous one reduces the risk } \\
\text { of infusion reactions and is more comfortable for the patient }\end{array}$ \\
\hline & & $\begin{array}{l}\text { - If toxicity occurs, interrupt the infusion and depending on the } \\
\text { severity of the infusion-related reactions, discontinuation of the } \\
\text { infusion or the use of glucocorticoids and antihistamines should } \\
\text { be considered }\end{array}$ \\
\hline
\end{tabular}

Infections

(especially:

hepatitis $B$,

C virus, Pneu-

mocystis jiroveci

pneumonia,

herpes zoster,

JC virus)

\author{
Pyrexia, asthenia, local \\ symptoms, neurological \\ symptoms of progressive \\ multifocal leukoencephalopathy
}

- Screening for hepatotropic viruses and prophylaxis should be performed before starting treatment

- Consider prophylaxis against pneumocystosis, herpes virus, screening for fungal infections if there are other risk factors such as concomitant use of fludarabine, alemtuzumab, immunosuppressants, or previous invasive fungal infection

- Optionally immunoglobulins can be given

- Asymptomatic or mild symptoms - clinical or diagnostic observations

- Moderate symptoms - oral antibiotics, antifungal or antiviral medications

- Severe symptoms - intravenous antibiotic, antifungal or antiviral intervention indicated 
Table 1 (cont.). The main adverse events of monoclonal antibodies (mAbs) used in therapy of hematological malignancies and prophylaxis and treatment suggestions

\begin{tabular}{|c|c|c|}
\hline Side effect & Clinical manifestation & Prophylaxis and treatment suggestions \\
\hline $\begin{array}{l}\text { Tumor lysis } \\
\text { syndrome }\end{array}$ & $\begin{array}{l}\text { Symptoms resulting from } \\
\text { hyperuricemia, hyperkalemia, } \\
\text { hyperphosphatemia, renal } \\
\text { failure, arrhythmias, convulsions }\end{array}$ & $\begin{array}{l}\text { - Prophylaxis - allopurinol and rehydration } \\
\text { - Treatment - hydration, use of hypouricemic drugs, renal } \\
\text { replacement therapy, correction of electrolyte disturbances }\end{array}$ \\
\hline $\begin{array}{l}\text { Cytokine release } \\
\text { syndrome }\end{array}$ & $\begin{array}{l}\text { Pyrexia, chills, hypotension, } \\
\text { tachypnea, fatigue, } \\
\text { cardiotoxicity }\end{array}$ & $\begin{array}{l}\text { - Mild symptoms - supportive treatment can be used } \\
\text { - Severe symptoms - treatment discontinuation, intravenous fluids, } \\
\text { vasopressors, glucocorticosteroids, and tocilizumab (an IL-6 inhibitor) }\end{array}$ \\
\hline $\begin{array}{l}\text { Hepatic veno-oc- } \\
\text { clusive disease/ } \\
\text { /hepatic sinusoi- } \\
\text { dal obstruction } \\
\text { syndrome }\end{array}$ & $\begin{array}{l}\text { Painful ascites, jaundice, weight } \\
\text { gain, hypoxia, edema, varicoses, } \\
\text { encephalopathy }\end{array}$ & $\begin{array}{l}\text { - Prophylactic administration of ursodeoxycholic acid in patients } \\
\text { treated with gemtuzumab ozogamicin at high risk of hepatotoxicity } \\
\text { - Optimization of intravascular volume with crystalloid or colloid } \\
\text { solutions (e.g. albumin) without fluid overload, colloid solutions } \\
\text { in the case of hypoalbuminemia } \\
\text { - Avoidance of nephrotoxic and hepatotoxic drugs } \\
\text { - High doses of glucocorticoids for moderate to severe cases } \\
\text { - In patients with clinically significant liver disease, as well as prior } \\
\text { hematopoietic cell transplantation, the benefits and risks of } \\
\text { administration of inotuzumab/gemtuzumab should be balanced } \\
\text { - Use lower, fractionated doses of both gemtuzumab and } \\
\text { inotuzumab } \\
\text { - Avoid conditioning regimens containing two alkylating agents } \\
\text { - An interval of at least } 3 \text { months between discontinuation } \\
\text { of gemtuzumab therapy and the hematopoietic cell transplant } \\
\text { - Defibrotide in patients with hepatic veno-occlusive disease } \\
\text { undergoing stem cell transplantation and optionally adding } \\
\text { high doses of glucocorticosteroids }\end{array}$ \\
\hline Cardiotoxicity & $\begin{array}{l}\text { Arrhythmias, changes in blood } \\
\text { pressure }\end{array}$ & $\begin{array}{l}\text { - ECG monitoring } \\
\text { - Serum electrolyte levels } \\
\text { - Withdrawal of drugs that interfere with heart rhythm } \\
\text { - Symptomatic treatment } \\
\text { - Withdrawal of medications that affect heart function } \\
\text { - Correction of electrolyte disturbances }\end{array}$ \\
\hline $\begin{array}{l}\text { Hematological } \\
\text { toxicity - } \\
\text { anemia, } \\
\text { leukopenia, } \\
\text { thrombo- } \\
\text { cytopenia }\end{array}$ & $\begin{array}{l}\text { Asthenia, infections, } \\
\text { hemorrhage/bleeding }\end{array}$ & $\begin{array}{l}\text { - Monitoring of complete blood count prior to drug administration } \\
\text { and performing physical examination and clinical tests for signs } \\
\text { and symptoms of infection, bleeding, hemorrhage, and other } \\
\text { symptoms of myelosuppression } \\
\text { - Dose delaying or permanent therapy discontinuation, and supportive } \\
\text { care are recommended to control myelosuppression } \\
\text { - Dithiothreitol may be helpful in preventing of daratumumab } \\
\text { binding to white blood cells }\end{array}$ \\
\hline Neurotoxicity & $\begin{array}{l}\text { Headache, tremors, confusion } \\
\text { or aphasia, convulsions or } \\
\text { dementia, changes in sensation, } \\
\text { movement }\end{array}$ & $\begin{array}{l}\text { - Mild symptoms - observation } \\
\text { - Moderate symptoms - discontinuation of therapy and } \\
\text { glucocorticosteroids } \\
\text { - Seizures grade } \geq 3-\text { treatment interruption and anti-epileptic } \\
\text { drugs recommended } \\
\text { - Mechanical hyperventilation, acetazolamide or mannitol }\end{array}$ \\
\hline $\begin{array}{l}\text { Autoimmune } \\
\text { reactions }\end{array}$ & $\begin{array}{l}\text { Selective red cell aplasia, } \\
\text { autoimmune hemolytic anemia, } \\
\text { immunological side effects in } \\
\text { multiple organs, most } \\
\text { commonly affecting the skin } \\
\text { and thyroid gland }\end{array}$ & $\begin{array}{l}\text { - In most cases the use of systemic steroids or other immunosu- } \\
\text { ppressants such as infliximab or mycophenolate } \\
\text { - Mild cutaneous toxic effects, grades } 1-2 \text { - topical emollients/ } \\
\text { /steroids and/or antihistamines, grade } 3 \text { - oral or intravenous } \\
\text { steroids and temporary treatment discontinuation, grade } 4 \text { is } \\
\text { a life-threatening condition associated with symptoms such as } \\
\text { erythema, purpura or epidermal detachment, requiring rapid } \\
\text { administration of intravenous steroids and permanent } \\
\text { discontinuation of therapy } \\
\text { - Blood glucose, electrolytes, TSH, and fT4 tests before each infusion, } \\
\text { - In the case of subclinical/symptomatic hypothyroidism, substitution } \\
\text { with thyroid hormones should be considered } \\
\text { - In symptomatic patients, especially with hyperthyroidism, } \\
\text { treatment with beta-blockers should be initiated, carbimazole } \\
\text { or steroids are rarely required }\end{array}$ \\
\hline
\end{tabular}

JC — John Cunningham; IL-6 - interleukin-6; ECG — electrocardiogram; TSH — thyroid-stimulating hormone; fT4 — free thyroxine 


\section{References}

1. Shah M, Rajha E, DiNardo C, et al. Adverse events of novel therapies for hematologic malignancies: what emergency physicians should know. Ann Emerg Med. 2020; 75(2): 264-286, doi: 10.1016/j.annemergmed.2019.07.015, indexed in Pubmed: 31561995.

2. Golay J, Taylor RP. The role of complement in the mechanism of action of therapeutic anti-cancer mAbs. Antibodies (Basel). 2020; 9(4), doi: 10.3390/antib9040058, indexed in Pubmed: 33126570.

3. Constantinescu C, Pasca S, Zimta AA, et al. Overview of the side-effects of FDA- and/or EMA-approved targeted therapies for the treatment of hematological malignancies. J Clin Med. 2020; 9(9), doi: 10.3390/jcm9092903, indexed in Pubmed: 32911829.

4. Salles G, Barrett M, Foà R, et al. Rituximab in B-cell hematologic malignancies: a review of 20 years of clinical experience. Adv Ther. 2017; 34(10): 2232-2273, doi: 10.1007/s12325-017-0612-x, indexed in Pubmed: 28983798.

5. Yelvington BJ. Subcutaneous rituximab in follicular lymphoma, chronic lymphocytic leukemia, and diffuse large B-cell lymphoma. J Adv Pract Oncol. 2018; 9(5): 530-534, indexed in Pubmed: 31086689.

6. Ruiz-Camps I, Aguilar-Company J. Risk of infection associated with targeted therapies for solid organ and hematological malignancies. Ther Adv Infect Dis. 2021; 8: 2049936121989548, doi: 10.1177/2049936121989548, indexed in Pubmed: 33680453.

7. Varma P, Falconer J, Aga A, et al. Rituximab-induced Crohnis disease. Scand J Gastroenterol. 2017; 52(5): 606-608, doi: 10.1080/00365521.2017.1280530, indexed in Pubmed: 28129697.

8. Urru SAM, Spila Alegiani S, Guella A, et al. Safety of switching between rituximab biosimilars in onco-hematology. Sci Rep. 2021; 11(1): 5956, doi: 10.1038/s41598-021-85563-1, indexed in Pubmed: 33727667.

9. Freeman CL, Sehn LH. A tale of two antibodies: obinutuzumab versus rituximab. Br J Haematol. 2018; 182(1): 29-45, doi: 10.1111/bjh.15232, indexed in Pubmed: 29741753.

10. Amitai I, Gafter-Gvili A, Shargian-Alon L, et al. Obinutuzumab-related adverse events: a systematic review and meta-analysis. Hematol Oncol. 2021; 39(2): 215-221, doi: 10.1002/hon.2828, indexed in Pubmed: 33252145.

11. Samuels C, Abbott D, Niemiec S, et al. Evaluation and associated risk factors for neutropenia with venetoclax and obinutuzumab in the treatment of chronic lymphocytic leukemia. Cancer Rep (Hoboken). 2021 [Epub ahead of print]: e1505, doi: 10.1002/ cnr2.1505, indexed in Pubmed: 34250757.

12. Gao S, Zhang M, Wu K, et al. Risk of adverse events in lymphoma patients treated with brentuximab vedotin: a systematic review and meta-analysis. Expert Opin Drug Saf. 2020; 19(5): 617-623, doi: 10.1080/14740338.2020.1718103, indexed in Pubmed: 31955620 .

13. Fargeot G, Dupel-Pottier C, Stephant M, et al. Brentuximab vedotin treatment associated with acute and chronic inflammatory demyelinating polyradiculoneuropathies. J Neurol Neurosurg Psychiatry. 2020; 91(7): 786-788, doi: 10.1136/jnnp-2020-323124, indexed in Pubmed: 32327451.

14. Makita S, Maruyama D, Tobinai K. Safety and efficacy of brentuximab vedotin in the treatment of classic Hodgkin lymphoma. Onco Targets Ther. 2020; 13: 5993-6009, doi: 10.2147/OTT.S193951, indexed in Pubmed: 32606807.

15. Moskowitz AJ. Optimizing the role of brentuximab vedotin in classical Hodgkin lymphoma therapy. Hematology Am Soc Hematol
Educ Program. 2018; 2018(1): 207-212, doi: 10.1182/asheducation-2018.1.207, indexed in Pubmed: 30504312.

16. Cheson BD, Bartlett NL, LaPlant B, et al. Brentuximab vedotin plus nivolumab as first-line therapy in older or chemotherapy-ineligible patients with Hodgkin lymphoma (ACCRU): a multicentre, single-arm, phase 2 trial. Lancet Haematol. 2020; 7(11): e808-e815, doi: 10.1016/S2352-3026(20)30275-1, indexed in Pubmed: 33010817.

17. Conde-Royo D, Juárez-Salcedo LM, Dalia S. Management of adverse effects of new monoclonal antibody treatments in acute lymphoblastic leukemia. Drugs Context. 2020; 9, doi: 10.7573/ dic.2020-7-2, indexed in Pubmed: 33110433

18. Sigmund AM, Sahasrabudhe KD, Bhatnagar B. Evaluating blinatumomab for the treatment of relapsed/refractory ALL: design, development, and place in therapy. Blood Lymphat Cancer. 2020; 10: 7-20, doi: 10.2147/BLCTT.S223894, indexed in Pubmed: 33173373.

19. Topp MS, Stein AS, Gökbuget N, et al. Blinatumomab as first salvage versus second or later salvage in adults with relapsed/ /refractory B-cell precursor acute lymphoblastic leukemia: Results of a pooled analysis. Cancer Med. 2021; 10(8): 2601-2610, doi: 10.1002/cam4.3731, indexed in Pubmed: 33734596.

20. Gavralidis A, Brunner AM. Novel therapies in the treatment of adult acute lymphoblastic leukemia. Curr Hematol Malig Rep. 2020; 15(4): 294-304, doi: 10.1007/s11899-020-00591-4, indexed in Pubmed: 32445026.

21. Li X, Zhou M, Qi J, et al. Efficacy and safety of inotuzumab ozogamicin (CMC-544) for the treatment of relapsed/refractory acute lymphoblastic leukemia and non-Hodgkin lymphoma: a systematic review and meta-analysis. Clin Lymphoma Myeloma Leuk. 2021; 21(3): e227-e247, doi: 10.1016/j.clml.2020.12.008, indexed in Pubmed: 33461955.

22. Corbacioglu S, Jabbour EJ, Mohty M. Risk factors for development of and progression of hepatic veno-occlusive disease/ /sinusoidal obstruction syndrome. Biol Blood Marrow Transplant. 2019; 25(7): 1271-1280, doi: 10.1016/j.bbmt.2019.02.018, indexed in Pubmed: 30797942.

23. Aujla A, Aujla R, Liu D. Inotuzumab ozogamicin in clinical development for acute lymphoblastic leukemia and non-Hodgkin lymphoma. Biomark Res. 2019; 7: 9, doi: 10.1186/s40364-019-0160-4, indexed in Pubmed: 31011424.

24. Muhamad NA, Mohd Dali NS, Mohd Yacob A, et al. Effect and safety of gemtuzumab ozogamicin for the treatment of patients with acute myeloid leukaemia: a systematic review protocol. BMJ Open. 2020; 10(6): e032503, doi: 10.1136/bmjopen-2019-032503, indexed in Pubmed: 32540885.

25. Pautas C, Raffoux E, Lambert J, et al. Fractionated doses of gemtuzumab ozogamicin with escalated doses of daunorubicin and cytarabine as first acute myeloid leukemia salvage in patients aged 50-70-year old: a phase 1/2 study of the acute leukemia French association. Am J Hematol. 2012; 87(1): 62-65, doi: 10.1002/ajh.22201, indexed in Pubmed: 22072535.

26. Molica M, Perrone S, Mazzone C, et al. CD33 Expression and gentuzumab ozogamicin in acute myeloid leukemia: two sides of the same coin. Cancers (Basel). 2021; 13(13), doi: 10.3390/ cancers13133214, indexed in Pubmed: 34203180.

27. Cortes JE, de Lima M, Dombret H, et al. Prevention, recognition, and management of adverse events associated with gemtuzumab ozogamicin use in acute myeloid leukemia. J Hematol Oncol. 2020; 13(1): 137, doi: 10.1186/s13045-020-00975-2, indexed in Pubmed: 33059764. 
28. Vollmar BS, Frantz C, Schutten MM, et al. Calicheamicin antibody-drug conjugates with improved properties. Mol Cancer Ther. 2021; 20(6): 1112-1120, doi: 10.1158/1535-7163.MCT-200035, indexed in Pubmed: 33722856.

29. Dima D, Dower J, Comenzo RL, et al. Evaluating daratumumab in the treatment of multiple myeloma: safety, efficacy and place in therapy. Cancer Manag Res. 2020; 12: 7891-7903, doi: 10.2147/ CMAR.S212526, indexed in Pubmed: 32904669.

30. Offidani M, Corvatta L, Morè S, et al. Daratumumab for the management of newly diagnosed and relapsed/refractory multiple myeloma: current and emerging treatments. Front Oncol. 2020; 10: 624661, doi: 10.3389/fonc.2020.624661, indexed in Pubmed: 33680948 .

31. Morè S, Petrucci MT, Corvatta L, et al. Monoclonal antibodies: leading actors in the relapsed/refractory multiple myeloma treatment. Pharmaceuticals (Basel). 2020; 13(12), doi: 10.3390/ ph13120426, indexed in Pubmed: 33260960.

32. Zhang T, Wang S, Lin T, et al. Systematic review and meta-analysis of the efficacy and safety of novel monoclonal antibodies for treatment of relapsed/refractory multiple myeloma. Oncotarget. 2017; 8(20): 34001-34017, doi: 10.18632/oncotarget.16987, indexed in Pubmed: 28454113.

33. Dimopoulos MA, Lonial S, Betts KA, et al. Elotuzumab plus lenalidomide and dexamethasone in relapsed/refractory multiple myeloma: Extended 4-year follow-up and analysis of relative progression-free survival from the randomized ELOQUENT-2 trial. Cancer. 2018; 124(20): 4032-4043, doi: 10.1002/cncr.31680, indexed in Pubmed: 30204239.

34. Ransohoff JD, Kwong BY. Cutaneous adverse events of targeted therapies for hematolymphoid malignancies. Clin Lymphoma Myeloma Leuk. 2017; 17(12): 834-851, doi: 10.1016/j. clml.2017.07.005, indexed in Pubmed: 28918995.

35. Sharma K, Janik JE, O>Mahony D, et al. Phase II study of alemtuzumab (CAMPATH-1) in patients with HTLV-1-associated adult T-cell leukemia/lymphoma. Clin Cancer Res. 2017; 23(1): 35-42, doi: 10.1158/1078-0432.CCR-16-1022, indexed in Pubmed: 27486175.

36. Alpdogan O, Kartan S, Johnson W, et al. Allogeneic hematopoietic stem cell transplantation in advanced stage mycosis fungoides and Sézary syndrome: a concise review. Chin Clin Oncol. 2019; 8(1): 12, doi: 10.21037/cco.2018.10.03, indexed in Pubmed: 30525754.

37. Wulf GG, Altmann B, Ziepert M, et al. ACT-2 study investigators. Alemtuzumab plus $\mathrm{CHOP}$ versus $\mathrm{CHOP}$ in elderly patients with peripheral T-cell lymphoma: the DSHNHL2006-1B/ACT-2 trial. Leukemia. 2021; 35(1): 143-155, doi: 10.1038/s41375-020-08385, indexed in Pubmed: 32382083.

38. Lenihan DJ, Alencar AJ, Yang D, et al. Cardiac toxicity of alemtuzumab in patients with mycosis fungoides/Sézary_syndrome.
Blood. 2004; 104(3): 655-658, doi: 10.1182/blood-2003-07-2345, indexed in Pubmed: 15073032.

39. Lloyd R, Nikolousis E, Kishore B, et al. Autoimmune cytopenias developing late post alemtuzumab-based allogeneic stem cell transplantation: presentation of short case series from a transplant center. Cell Transplant. 2020; 29: 963689720950641, doi:_10.1177/0963689720950641, indexed in Pubmed: 32806929 .

40. Ollila TA, Sahin I, Olszewski AJ. Mogamulizumab: a new tool for management of cutaneous T-cell lymphoma. Onco Targets Ther. 2019; 12: 1085-1094, doi: 10.2147/OTT.S165615, indexed in Pubmed: 30799938.

41. Blackmon AL, Pinter-Brown L. Spotlight on mogamulizumabKpkc for use in adults with relapsed or refractory mycosis fungoides or Sézary syndrome: efficacy, safety, and patient selection. Drug Des Devel Ther. 2020; 14: 3747-3754, doi: 10.2147/DDDT. S185896, indexed in Pubmed: 32982179.

42. Moore DC, Elmes JB, Shibu PA, et al. Mogamulizumab: sn snti-CC chemokine receptor 4 antibody for T-cell lymphomas. Ann Pharmacother. 2020; 54(4): 371-379, doi: 10.1177/1060028019884863, indexed in Pubmed: 31648540.

43. Alatrash G, Daver N, Mittendorf EA. Targeting immune checkpoints in hematologic malignancies. Pharmacol Rev. 2016; 68(4): 1014-1025, doi: 10.1124/pr.116.012682, indexed in Pubmed: 27664133.

44. Matsuki E, Younes A. Checkpoint inhibitors and other immune therapies for Hodgkin and non-Hodgkin lymphoma. Curr Treat Options Oncol. 2016; 17(6): 31, doi: 10.1007/s11864-016-0401-9, indexed in Pubmed: 27193488.

45. Hradska K, Hajek R, Jelinek T. Toxicity of immune-checkpoint inhibitors in hematological malignancies. Front Pharmacol. 2021; 12: 733890, doi: 10.3389/fphar.2021.733890, indexed in Pubmed: 34483944 .

46. Bewersdorf JP, Stahl M, Zeidan AM. Immune checkpoint-based therapy in myeloid malignancies: a promise yet to be fulfilled. Expert Rev Anticancer Ther. 2019; 19(5): 393-404, doi: 10.1080/14737140.2019.1589374, indexed in Pubmed: 30887841.

47. Stahl M, Goldberg AD. Immune checkpoint inhibitors in acute myeloid leukemia: novel combinations and therapeutic targets. Curr Oncol Rep. 2019; 21(4): 37, doi: 10.1007/s11912-019-0781-7, indexed in Pubmed: 30904967.

48. Usmani SZ, Schjesvold F, Oriol A, et al. KEYNOTE-185 Investigators. Pembrolizumab plus lenalidomide and dexamethasone for patients with treatment-naive multiple myeloma (KEYNOTE-185): a randomised, open-label, phase 3 trial. Lancet Haematol. 2019; 6(9): e448-e458, doi: 10.1016/S2352-3026(19)30109-7, indexed in Pubmed: 31327689. 
